\title{
Assessing the effectiveness of role assignment on improving student's asynchronous online discussion participation
}

\begin{abstract}
Taking into account prior research suggesting a lack of student participation in online discussions, this study examines the influence of peer moderator (PM) role assignment on students' participation and that of their peers' participation in online discussions. Eighty-four participants operated in a moderator role, reciprocally. Moreover, the study examines the differences in the level of e-moderation supports enacted by PMs of high-and low-density online discussions. Online participation was assessed using log files of seven-week discussions and social network analysis techniques. Quantitative content analysis was applied with online interaction transcripts of PMs for two groups of online discussions. The results indicated that students in the PM role reached significantly higher level of participation quantity and patterns and their non-posting participation significantly influenced all indicators of group participation. Further, high-and low-density online discussions differed significantly with regards to frequency of PMs' e-moderation supports.
\end{abstract}

Keyword: Online discussions; Participation; Peer moderation; Quantitative content analysis; Social network analysis 